\title{
Pressure based MRI-compatible muscle fascicle length and joint angle estimation
}

\author{
Hyungeun Song ${ }^{1,2}$ (D) Erica Israel ${ }^{1,3}$, Shriya Srinivasan ${ }^{1,2}$ and Hugh Herr ${ }^{1,3^{*}}$
}

\begin{abstract}
Background: Functional magnetic resonance imaging (fMRI) provides critical information about the neurophysiology of the central nervous systems (CNS), posing clinical significance for the understanding of neuropathologies and advancement of rehabilitation. Typical fMRI study designs include subjects performing designed motor tasks within specific time frames, in which fMRI data are then analyzed by assuming that observed functional brain activations correspond to the designed tasks. Therefore, developing MRI-compatible sensors that enable real-time monitoring of subjects' task performances would allow for highly accurate fMRI studies. While several MRI-compatible sensors have been developed, none have demonstrated the ability to measure individual muscle fascicle length during $\mathrm{fMRI}$, which could help uncover the complexities of the peripheral and central nervous systems. Furthermore, previous MRI-compatible sensors have been focused on biologically intact populations, limiting accessibility to populations such as those who have undergone amputation.
\end{abstract}

Methods: We propose a lightweight, low-cost, skin impedance-insensitive pressure-based muscular motion sensor (pMMS) that provides reliable estimates of muscle fascicle length and joint angle. The muscular motions are captured through measured pressure changes in an air pocket wrapped around the muscle of interest, corresponding to its muscular motion. The muscle fascicle length and joint angle are then estimated from the measured pressure changes based on the proposed muscle-skin-sensor interaction dynamics. Furthermore, we explore an integration method of multiple pMMS systems to expand the sensor capacity of estimating muscle fascicle length and joint angle. Ultrasound imaging paired with joint encoder measurements are utilized to assess pMMS estimation accuracy of muscle fascicle length in the tibialis anterior (TA) and ankle joint angle, respectively, of five biologically intact subjects.

Results: We found that a single pMMS sufficiently provides robust and accurate estimations of TA muscle fascicle length and ankle joint angle during dorsiflexion at various speeds and amplitudes. Further, differential pressure readings from two pMMSs, in which each pMMS were proximally and distally placed, were able to mitigate errors due to perturbations, expanding pMMS capacity for muscle fascicle length and ankle joint angle estimation during the full range of plantar flexion and dorsiflexion.

Conclusions: Our results from this study demonstrate the feasibility of the pMMS system to further be incorporated in fMRI settings for real-time monitoring of subjects' task performances, allowing sophisticated fMRI study designs.

Keywords: Wearable sensor, Muscle state sensor, MRI-compatible

*Correspondence: hherr@media.mit.edu

${ }^{1}$ Center for Extreme Bionics, Massachusetts Institute of Technology (MIT)

Media Lab, 02139 Cambridge, MA, USA

${ }^{3}$ Department of Media Arts and Sciences, MIT, 02139 Cambridge, MA, USA

Full list of author information is available at the end of the article

(c) The Author(s). 2020 Open Access This article is licensed under a Creative Commons Attribution 4.0 International License, which permits use, sharing, adaptation, distribution and reproduction in any medium or format, as long as you give appropriate credit to the original author(s) and the source, provide a link to the Creative Commons licence, and indicate if changes were made. The images or other third party material in this article are included in the article's Creative Commons licence, unless indicated otherwise in a credit line to the material. If material is not included in the article's Creative Commons licence and your intended use is not permitted by statutory regulation or exceeds the permitted use, you will need to obtain permission directly from the copyright holder. To view a copy of this licence, visit http://creativecommons.org/licenses/by/4.0/. The Creative Commons Public Domain Dedication waiver (http://creativecommons.org/publicdomain/zero/1.0/) applies to the data made available in this article, unless otherwise stated in a credit line to the data. 


\section{Background}

Functional magnetic resonance imaging (fMRI) is utilized for investigating brain activity in subjects performing given tasks within specific time frames during a functional scan [1]. fMRI allows for a consecutive time-series of brain activity to be compared with specific tasks and movements, which is critical data for uncovering the connections between motor dysfunction and neural activity. To synchronize fMRI frames with motor task data, and to enable real-time monitoring of subjects' task performances, various MRI-compatible sensors have been proposed to measure grip force [2], joint position [3-5], and net torque [6-9]. Meanwhile, the exchange of efferent motor signals from the brain and afferent sensory signals from the muscular system, through mechanoneural transduction within muscles, is known to play a critical role in fine motor control $[10,11]$. Therefore, the development of a MRI-compatible sensor that provides muscle fascicle length would allow for the assessment of afferent signaling within muscles. Furthermore, for populations such as persons who have undergone amputation, assessment of the subjects' task performances needs to be based on muscle fascicle movements, independent of joint kinematics and kinetics. Thus, the implementation of a MRI-safe sensor for collecting muscle fascicle data in conjunction with brain activity may be immensely important for advancing our understanding of the complex network between the central and peripheral nervous systems, and evaluating the efficacy of rehabilitation strategies to restore neuromuscular control.

Implantable sonomicrometery crystals have been reported to accurately record real time muscle fascicle length changes. Researchers have used sonomicrometery crystals to track muscle fascicle length and dynamics through direct measurement of crystal activity using a sonomicrometry amplifier [12]. Recently, it has been discovered that magnets can be used in a similar manner. Magnetic fields are able to pass through biological tissues which allows for muscle fascicle length changes to be recorded, via distance tracking between implantable magnets, without a powered system [13]. These methods have been proven successful for highly accurate muscular tracking without being affected by outside noise or skin impedance. However, both require invasive surgery and are strictly limited to a majority of the biologically intact population. Also, magnet tracking still needs to be tested for long term viability in human applications as well as MRI-compatibility.

Sonomyography via ultrasound imaging has also been utilized to derive muscle fascicle length and pennation angle measurements. This has been successful in characterizing muscle architecture for applications including gait abnormality detection, quantifying the dynamics of individual muscles, and measuring proprioceptive intent
$[14,15]$. An advantage of ultrasound imaging is its ability to isolate muscles of interest, down to individual fascicles, resulting in precise, easy-to-visualize measurements. However, this modality is bound to clinical and laboratory settings, and the quality of the imaging is limited to trained technicians. The reliability of ultrasound imaging may also be diminished due to its reliance on individual interpretation of a given image [16].

For the purpose of avoiding the aforementioned, several efforts have focused on cross-sectional expansion of muscle during its movements $[17,18]$. These muscle movement-based methodologies place an air pocket, or force sensitive registers (FSRs), on the muscle of interest, and by tracking cross-sectional expansion via air pressure or contact forces, the muscle activity was estimated. Force myography (FMG) has also been explored for detecting volumetric changes of specific muscles inside a compression garment with the intention of estimating muscle activity [19]. These systems have advantages in terms of practicality for being insensitive to skin impedance changes, and do not require invasive attachment to the skin. In the previously mentioned studies, however, the general estimation strategies of variables such as muscle fascicle length and joint angles, that have non-negligible non-linearity between the sensor outputs and target variable, were not explored. In addition, these studies have neglected muscle-skin interactions and sensor dynamics, e.g. air pocket dynamics and non-linearity of FSRs.

Although most of the sensors mentioned above have proved successful in clinical settings, they remain futile in conjunction with fMRI for safety and logistical reasons. In this paper, we propose a MRI-safe, non-invasive, cost efficient, pressure-based muscular motion sensor (pMMS) and associated methodology. The pMMS accounts for muscle-skin-sensor dynamics and addresses the ability to estimate changes in muscle fascicle length and joint angles in free-space. Similar to the methodology of the previously mentioned air pocket system, the pMMS utilizes pressure changes in the air pocket due to the cross-sectional expansion of the muscle as shown in Fig. 1a. The proposed method includes a general estimation strategy of target variables that have non-negligible non-linearity with the sensor outputs, to provide robust, real-time estimates of muscle fascicle length and joint angle. These estimations from the pMMS are evaluated by comparative experiments using ultrasound imaging and a joint encoder. Through our investigation, we demonstrate the device's capability to reject perturbations from surrounding muscles by utilizing the differential pressure of two pMMSs (differential-pMMS), and demonstrate pMMSs' muscle fascicle length and joint angle estimation accuracy during the full range of motion of the ankle joint. The pMMS may provide several clinically-significant contributions including the ability to synchronize muscle fascicle length and 


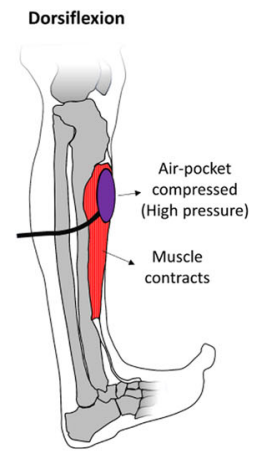

(a)

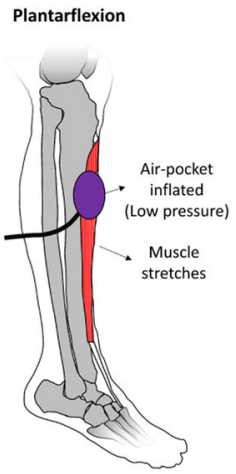

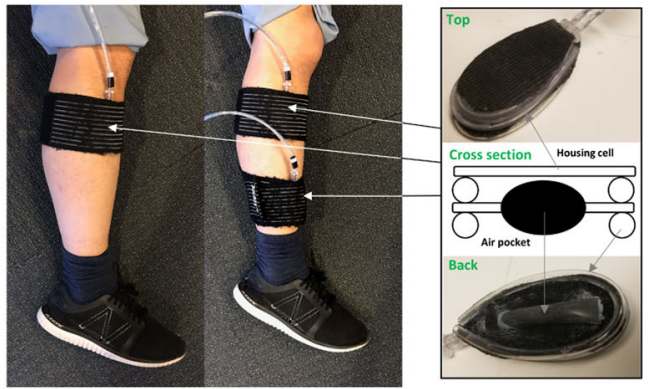

(b)

Fig. 1 A pressure-based muscular movement sensor (pMMS). The pMMS measures the cross-sectional expansion of the muscle via pressure changes in the air pocket. a Mechanism of pMMS, b implementation of single-pMMS (left) and differential-pMMS (right)

joint angle data with fMRI data in real time while ensuring user comfort and affordability.

\section{Methods}

\section{Mechanism and design of the pressure-based muscular movement sensor (pMMS)}

The pressure-based muscular motion sensor (pMMS) consists of a small air pocket $(3 \times 0.8 \times 0.8 \mathrm{~cm})$ and a housing cell as shown in Fig. 1b. When a muscle is shortened or lengthened, the cross-sectional area of the muscle varies due to changes in the overlapping area of myosin and actin filaments [20], which are biological structures for muscular force production, and muscle fiber pennation angle [21]. By measuring pressure changes within the pMMS wrapped around a muscle of interest, the pMMS reading correlates to the cross-sectional expansion of the muscle, which allows for estimation of the target variables, muscle fascicle length and joint angle, based on the sensor dynamics as shown in Fig. 2a. The housing cell incorporates a double layer structure to minimize pressure changes due to external perturbations and isolates the muscle of interest from surrounding muscles as shown in Fig. 1.

\section{Modeling}

The schematic diagram of pMMS dynamics is shown in Fig. 2a. In this work, an internal equilibrium point $x_{\mathrm{m}}$ is assumed, which has a static non-linear relationship $g($.) between its variance $\delta x_{\mathrm{m}}$ and that of the target variable $\delta \eta$. $x_{\mathrm{m}}$ is utilized to reflect the $\eta$-dependent steady pressure of the air pocket $P_{\mathrm{a}}$. Overall, $\delta \eta$ results $\delta x_{\mathrm{m}}$ based on $g($.$) ,$ which propagates through the muscle-skin and deforms

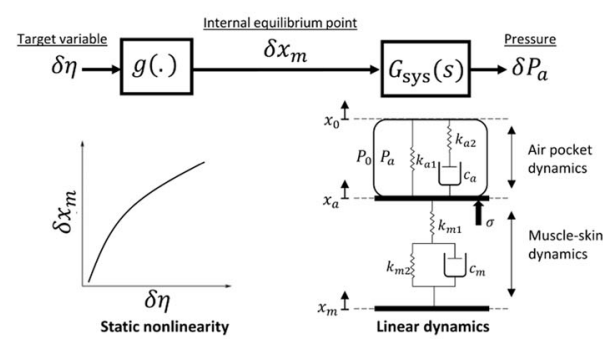

(a)

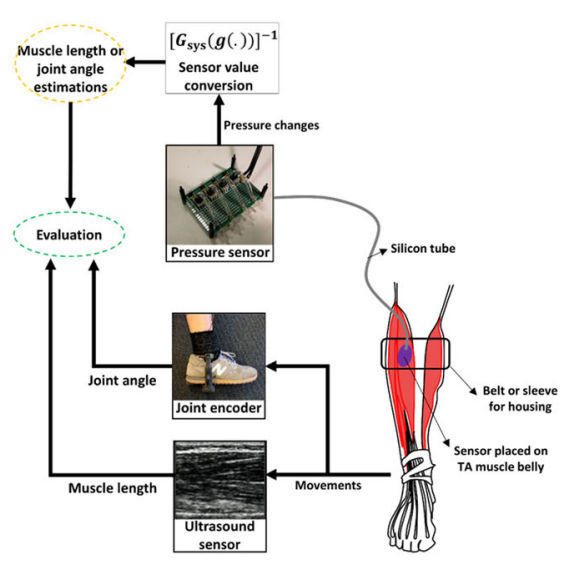

(b)

Fig. 2 Schematic diagram of pMMS dynamics and evaluation procedures. The pMMS is placed on the tibialis anterior (TA) muscle and secured in place by a bandage or sleeve. The pMMS outputs are converted to the target variable (muscle fascicle length or joint angle) based on the identified muscle-skin-pMMS dynamics consisting of a static non-linearity and a linear system. The performance of pMMS is evaluated by the joint encoder and ultrasound sensor. a pMMS dynamics, b block diagram of experimental setup 
the air pocket, causing pressure changes inside of the air pocket $\delta P_{\mathrm{a}}$. The pMMS dynamics are derived as follows.

From the ideal gas law, $P_{\mathrm{a}}$ is described as

$$
P_{\mathrm{a}}=\frac{n R T}{A\left(x_{0}-x_{\mathrm{a}}\right)}
$$

where $n, R$, and $T$ indicates the moles of gas in the air pocket, the gas constant, the absolute temperature, respectively, and $A, x_{0}$, and $x_{\mathrm{a}}$ are the contact area, positions of top and bottom of the air pocket, respectively. By taking the derivative of Eq.1, we obtain

$$
\begin{aligned}
\delta P_{\mathrm{a}} & =-\frac{n R T}{A\left(x_{0}-x_{\mathrm{a}}\right)^{2}} \delta\left(x_{0}-x_{\mathrm{a}}\right) \\
& =-K_{\mathrm{p}} \delta\left(x_{0}-x_{\mathrm{a}}\right)
\end{aligned}
$$

where $K_{\mathrm{p}}$ indicates $\frac{n R T}{A\left(x_{0}-x_{\mathrm{a}}\right)^{2}}$. Because $A$ and $n$ were constant by the design of pMMS and $T$ reached equilibrium with body temperature during trials, $K_{\mathrm{p}}$ was considered as constant for marginal changes of $\delta\left(x_{0}-x_{\mathrm{a}}\right)$. The viscoelastic properties of the air pocket are known to be well-described by two springs and a damper [22, 23], which can be described using the force balance equation as

$$
\sigma A=A\left(P_{\mathrm{a}}-P_{0}\right)-\left(k_{\mathrm{a} 1}+\frac{k_{\mathrm{a} 2} c_{\mathrm{a}} s}{k_{\mathrm{a} 2}+c_{\mathrm{a}} s}\right)\left(x_{0}-x_{\mathrm{a}}\right)
$$

where $\sigma$ and $P_{0}$ indicate the contact stress between pMMS and skin surface and barometric pressure, respectively. $k_{\mathrm{a} 1}, k_{\mathrm{a} 2}$, and $c_{\mathrm{a}}$ are the elastic and damping components of the pMMS, respectively. From Eq. $2 \mathrm{~b}$ and the derivative of Eq. 3 with respect to time, the dynamics between $\sigma$ and $P_{\mathrm{a}}$ can be described as

$$
\begin{aligned}
\delta \sigma & =\delta P_{\mathrm{a}}-\frac{1}{A}\left(k_{\mathrm{a} 1}+\frac{k_{\mathrm{a} 2} c_{\mathrm{a}} s}{k_{\mathrm{a} 2}+c_{\mathrm{a}} s}\right) \delta\left(x_{0}-x_{\mathrm{a}}\right) \\
& =G_{\mathrm{s}}(s) \delta P_{\mathrm{a}}
\end{aligned}
$$

where $G_{\mathrm{s}}(s)$ indicates $1+\frac{1}{A K_{\mathrm{p}}}\left(k_{\mathrm{a} 1}+\frac{k_{\mathrm{a} 2} c_{\mathrm{a}} s}{k_{\mathrm{a} 2}+c_{\mathrm{a}} s}\right)$. Additionally, muscle-skin dynamics are known to have three distinguishable mechanical behaviors: the pure elastic, viscoelastic, and constant creep phase [24, 25]. However, the constant creep phase only becomes dominant when deformation of the muscle-skin remains constant for long periods, which is difficult to be performed by voluntary muscle contraction. This high order property is further regressed by air pocket dynamics, thereby we assume that the constant creep phase is negligible. Then, the muscleskin dynamics are described by an elastic component, $k_{\mathrm{m} 1}$, serially connected to another elastic component, $k_{\mathrm{m} 2}$, and a damper, $c_{\mathrm{m}}$, to address the pure elastic and viscoelastic phases, or

$$
\begin{aligned}
\delta \sigma & =\frac{1}{A} \frac{k_{\mathrm{m} 1}\left(k_{\mathrm{m} 2}+c_{\mathrm{m}} s\right)}{\left(k_{\mathrm{m} 1}+k_{\mathrm{m} 2}+c_{\mathrm{m}} s\right)} \delta\left(x_{\mathrm{a}}-x_{\mathrm{m}}\right) \\
& =G_{\mathrm{m}}(s)\left(\frac{1}{K_{\mathrm{p}}} \delta P_{\mathrm{a}}+\delta\left(x_{0}-x_{\mathrm{m}}\right)\right)
\end{aligned}
$$

where $G_{\mathrm{m}}(s)$ indicates $\frac{1}{A} \frac{k_{\mathrm{m} 1}\left(k_{\mathrm{m} 2}+c_{\mathrm{m}} s\right)}{\left(k_{\mathrm{m} 1}+k_{\mathrm{m} 2}+c_{\mathrm{m}} s\right)}$ and $x_{\mathrm{m}}$ represents the $\eta$-dependent internal equilibrium point of the muscleskin dynamics. Eq. 5b was derived by the combination of Eq. $2 \mathrm{~b}$ and $5 \mathrm{a}$, putting $x_{\mathrm{a}}-x_{\mathrm{m}}=-\left(x_{0}-x_{\mathrm{a}}\right)+x_{0}-x_{\mathrm{m}}$. Then, from Eq. $4 \mathrm{~b}$ and $5 \mathrm{~b}$, the overall muscle-skin-pMMS dynamics are described as

$$
\begin{aligned}
\delta P_{\mathrm{a}} & =\frac{K_{\mathrm{p}} G_{\mathrm{m}}(s)}{G_{\mathrm{m}}(s)-K_{\mathrm{p}} G_{\mathrm{s}}(s)} \delta\left(x_{\mathrm{m}}-x_{0}\right) \\
& =G_{\text {sys }}(s) \delta\left(x_{\mathrm{m}}-x_{0}\right) .
\end{aligned}
$$

Because the air pocket is shielded by a double layer housing cell, the external perturbations to $\delta P_{\mathrm{a}}$, e.g. the muscular movements of surrounding muscles, and external forces, are negligible, i.e. $\delta x_{0}=0$. To enhance generality of the proposed model, the $\eta-x_{\mathrm{m}}$ relationship is not restricted by the linearity assumption; the static nonlinearity $g($.$) is assumed between \delta x_{\mathrm{m}}$ and $\delta \eta$, or

$$
\delta x_{\mathrm{m}}=g(\delta \eta) .
$$

Then, as shown in Fig. $2 \mathrm{a}$, the $\eta-P_{\mathrm{a}}$ relationship is described as

$$
\delta P_{\mathrm{a}}=G_{\text {sys }}(s) g(\delta \eta) .
$$

Therefore, $\delta \eta$ can be estimated from $\delta P_{\mathrm{a}}$ as

$$
\delta \eta=g^{-1}\left(G_{\text {sys }}^{-1}(s) \delta P_{\mathrm{a}}\right) .
$$

Note that the inverse model of pMMS dynamics for the variable estimation can be described as the linear system $G_{\text {sys }}^{-1}$ and the static non-linearity property $g^{-1}($.$) , or the$ Wiener system.

\section{System identification}

In this work, the system identification of pMMS is conducted by the revision of the Wiener system identification methodology addressed in [26]. Note that, because both $G_{\mathrm{s}}$ and $G_{\mathrm{m}}$ are first order dynamics with a single zero (Eq. 6a), $G_{\text {sys }}^{-1}(s)$ is described as a second order system with two zeros, or

$$
G_{\text {sys }}^{-1}(s)=\frac{a_{2} s^{2}+a_{1} s+a_{0}}{s^{2}+b_{1} s+b_{0}}
$$

where $a_{\mathrm{i}}$ and $b_{\mathrm{j}}$ indicate coefficients. Then, the linear dynamics $G_{\text {sys }}^{-1}(s)$ and static non-linearity $g^{-1}($.) of Eq. 9 are identified by the following process:

1. Estimate the best fit for the linear dynamics $\hat{G}_{\text {sys }}^{-1}(s)$ from $\delta P_{\mathrm{a}}$ and $\delta \eta$.

2. Estimate output of the linear dynamics $\hat{y}$ from the convolution of $\hat{G}_{\text {sys }}^{-1}(s)$ with $\delta P_{\mathrm{a}}$.

3. Fit a high-order polynomial $g_{\mathrm{p}}^{-1}($.) between $\hat{y}$ and $\delta \eta$. 
4. Re-estimate $\hat{y}$ from $\eta$ via the inverse of the polynomial

5. Re-estimate $\hat{G}_{\text {sys }}^{-1}(s)$ from $\delta P_{\mathrm{a}}$ and $\hat{y}$.

6. Re-estimate $\hat{y}$ from the convolution of $\hat{G}_{\text {sys }}^{-1}(s)$ with $\delta P_{\mathrm{a}}$.

7. Go to the step (3) (another iteration) or go to the step (8) (final estimation)

8. Create lookup table $g_{1}^{-1}($.$) to model high order$ nonlinearity between $g_{\mathrm{p}}^{-1}(\hat{y})$ and $\delta \eta$.

Note that the identified static non-linearity $\hat{g}^{-1}$ is determined as $g_{\mathrm{l}}^{-1}\left(g_{\mathrm{p}}^{-1}().\right)$. In addition, because $\delta P_{\mathrm{a}}$ has strong correlations with the muscle fascicle length $l_{\mathrm{m}}$ and joint angle $\theta$, we assume a second order polynomial for $g_{\mathrm{p}}($. during iterative system identification procedure.

\section{Model verification of $\mathrm{pMMS}$}

The pMMS estimation of muscle fascicle length and joint angle is evaluated by comparing fascicle length and joint angle measurements via the ultrasound sensor and joint encoder as shown in Fig. $2 \mathrm{~b}$. The proposed model is then verified by measuring muscle motion of the tibialis anterior (TA). Five subjects (S1-S5) participated in this study as shown in Table 1. All experiments were carried out with informed consent at the Massachusetts Institute of Technology (MIT), under the approval of the Committee on the Use of Humans as Experimental Subjects (COUHES). The pMMS was affixed to the skin overlying the muscle using a compression sleeve or elastic wrap depending on the calf circumference and comfort of each subject. The pMMS placement was optimized to maximize the pressure sensor outputs during dorsiflexion. For system identification and evaluation of the pMMS estimations of the joint angle $\theta$ and muscle fascicle length $l_{\mathrm{m}}$, the joint encoder and ultrasound sensor were utilized as shown in Fig. 2b. For all trials, the subjects were asked to wear the joint encoder on their ankle to have the measurements of $\theta$ correspond to the pMMS outputs. Due to practical constraints surrounding ultrasound sensor placement alongside the pMMS, $\delta l_{\mathrm{m}}$ is calculated from $\delta \theta$ values based on a lookup table capturing $\theta$ and $l_{\mathrm{m}}$ which were identified prior to the trials without the pMMS. In addition, the $\delta l_{\mathrm{m}}$ was normalized by the full range of $\delta l_{\mathrm{m}}$ to evaluate over different individuals, referred to as $\Delta \epsilon_{\mathrm{m}}$.

Table 1 Subject descriptions

\begin{tabular}{llll}
\hline Subject & Gender & Height & Weight \\
\hline S1 & Male & $173 \mathrm{~cm}$ & $76 \mathrm{~kg}$ \\
S2 & Female & $165 \mathrm{~cm}$ & $40 \mathrm{~kg}$ \\
S3 & Female & $167 \mathrm{~cm}$ & $48 \mathrm{~kg}$ \\
S4 & Male & $196 \mathrm{~cm}$ & $100 \mathrm{~kg}$ \\
S5 & Male & $188 \mathrm{~cm}$ & $98 \mathrm{~kg}$ \\
\hline
\end{tabular}

To validate the proposed pMMS model, patients were asked to dorsiflex their foot, activating only the TA, from neutral position to full dorsiflexion. Full range of motion (ROM) that involves full plantar flexion would have introduced external perturbations from surrounding muscles into the measurement which will be detailed in the later portion of this report. In the first experiment, half-amplitude sweep trials, subjects were asked to dorsiflex at approximately 30,60, and 90 percent of their full range of dorsiflexion at a steady pace. However, no specific joint trajectory was provided for the subjects to allow variance in the movements between each cycle. This range of movement enabled us to fit system parameters across a larger range of muscle movements. For the second experiment, half-frequency sweep trials, the subjects repeatedly performed dorsiflexion in the following pattern of paces: slow, fast, and hold; in which they stopped at full dorsiflexion for at least 3 seconds. Similar to the halfamplitude sweep trials, no specific trajectory guidelines were given to the subjects. Two data sets of each experimental scheme were collected. The system identification was conducted based on an integrated data set which consists one of two data set of both half-amplitude and half-frequency sweep trials. Model verification was conducted by comparing the estimates of the target variables, based on the identified system model, to their measured values of the trials that were not used in the system identification procedures.

\section{External perturbations from surrounding muscles}

In the previous experimental scheme, the pMMS model was evaluated under a limited ROM (half-amplitude and half-frequency sweep trials) to minimize external perturbations and focus on the sensor model verification. However, in practice, the muscular movements of surrounding muscles, e.g. peroneus longus (PL), tibialis posterior (TP), and antagonist muscles, e.g. gastrocnemius (GAS), may not be negligible and cause large estimation errors particularly at the extreme ends of ROM. A typical pMMS readings during full dorsiflexion and plantar flexion is demonstrated in Fig. 3a. During extreme plantar flexion, the pressure values do not uniformly correlate with the muscular movements due to external perturbations, which are marked as asterisks $(*)$. For these values, because the pMMS cannot recognize whether the current pressure readings correspond to a small dorsiflexion or extreme plantar flexion, the external perturbations could cause significant errors in the both system identification and variable estimation as shown in Fig. 3c-f.

To investigate the effect of these external perturbations, all five subjects (S1-S5) were asked to perform two types of full range dorsiflexion and plantar flexion: full-amplitude sweep and full-frequency sweep trials. During the full-amplitude sweep trials, the subjects 


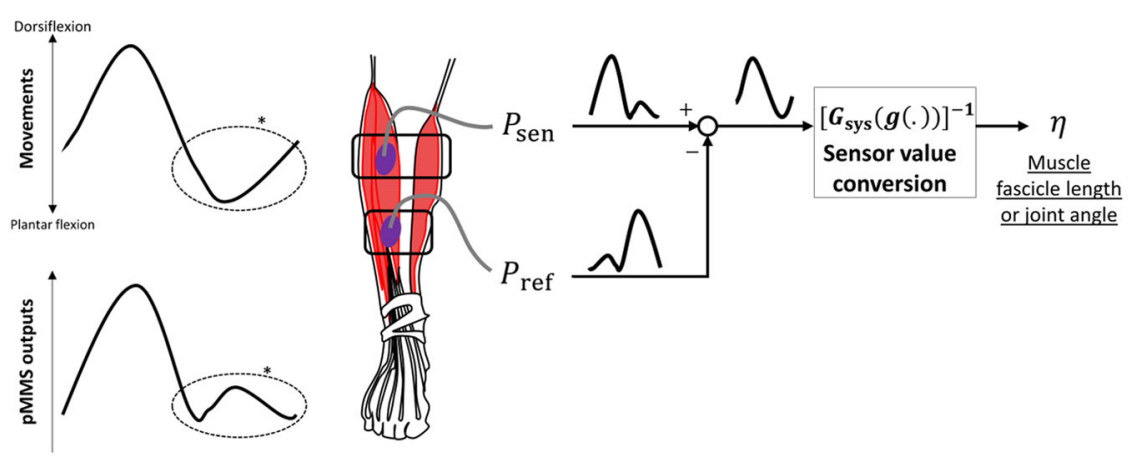

(a)
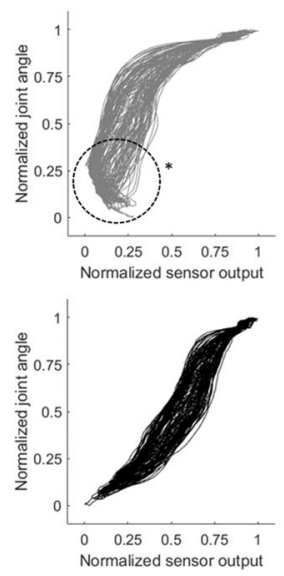

(c)
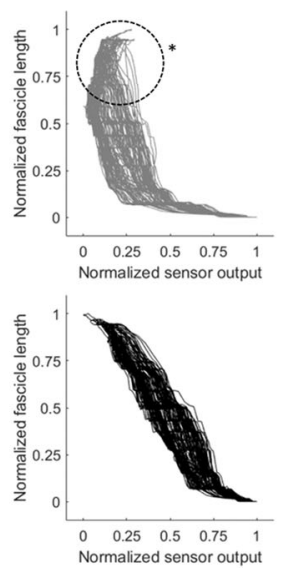

(d)
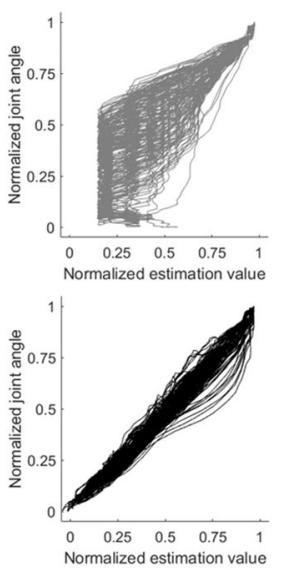

(e)

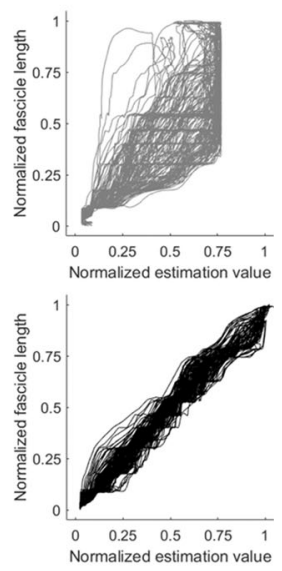

(f)

Fig. 3 External perturbations to the pMMS at extreme range of motion (ROM). The pMMS outputs do not uniformly correlate with the muscular movements at extreme ROM (circled with *). These perturbations are compensated by a differential-pMMS. a Typical single-pMMS outputs from the TA during dorsiflexion and plantar flexion, b schematic diagram of the differential-pMMS, c representatives of singe-pMMS (top) and differential-pMMS (bottom) outputs to varying joint angles, $\mathbf{d}$ representatives of singe-pMMS (top) and differential-pMMS (bottom) outputs to varying muscle fascicle length e representative linear plot of singe-pMMS (top) and differential-pMMS (bottom) estimation of varying joint angles, $\mathbf{f}$ representative linear plot of singe-pMMS (top) and differential-pMMS (bottom) estimation of varying muscle fascicle length

repeatedly performed dorsiflexion and plantar flexion, gradually increasing the displacement from neutral position with each cycle. For the full-frequency sweep trials, the subjects were asked to repeat the same task as the half frequency sweep trial but with their full ROM (full dorsiflexion to full plantar flexion). No specific joint trajectory was given for any trials to allow their dorsiflexion and plantar flexion to be varied. At least two trials for each type of task were obtained for system identification and validation. The system identification and data processing were conducted in a similar manner to the previously described method.

\section{External perturbation compensation by differential-pMMS}

To compensate external perturbations from surrounding muscles, we use an additional pMMS as a reference value, and utilize the differential pressure of two pMMSs (differential-pMMS) to amplify the pressure changes by the muscular movement of interest and cancel out external perturbations as shown in Fig. 3b. This provides uniform positive or negative correlation with the target variables as shown in Fig. 3c-f. Thus, for the muscles that are affected by non-negligible external perturbations, the differential pressure $\delta P_{\text {diff }}$ can be used as

$$
\begin{aligned}
\delta P_{\text {diff }} & =\delta P_{\text {sen }}-\delta P_{\text {ref }} \\
\delta \eta & =g^{-1}\left(G_{\text {sys }}^{-1}(s) \delta P_{\text {diff }}\right) .
\end{aligned}
$$

to estimate $\delta \eta$ where $\delta P_{\text {sen }}$ and $\delta P_{\text {ref }}$ indicate the pressure values of the pMMS on the muscle belly and reference point, respectively.

\section{Results}

\section{Identified system dynamics}

The identified linear system dynamics, $G_{\text {sys }}^{-1}(s)$, are given in Table 2. See Supplemental information for subjectspecific dynamics. Large variances were found in the 
Table 2 Identified linear system dynamics, $G_{\text {sys }}^{-1}(s)$, from half-amplitude and half-frequency (H.H.) and full-amplitude and full-frequency (F.F.) trials for joint angle (J.A.) and fascicle length (F.L.) estimations

\begin{tabular}{llllll}
\hline Type & $a_{2}$ & $a_{1}$ & $a_{0}$ & $b_{1}$ & $b_{0}$ \\
\hline H.H.J.A. (single) & $165.5 \pm 29.8$ & $1823 \pm 1587$ & $1836 \pm 1807$ & $9.7 \pm 8.5$ & $8.5 \pm 8.3$ \\
F.F.J.A. (single) & $140.2 \pm 56.6$ & $5115 \pm 5049$ & $682.2 \pm 578.0$ & $11.6 \pm 10.2$ & $8.6 \pm 5.6$ \\
F.F.J.A. (differential) & $143.9 \pm 53.5$ & $7189 \pm 2359$ & $145.5 \pm 87.7$ & $26.1 \pm 8.7$ & $1.3 \pm 0.8$ \\
H.H.F.L. (single) & $-47.7 \pm 19.6$ & $-202.4 \pm 178.6$ & $-183.5 \pm 161.4$ & $2.5 \pm 2.0$ & $1.8 \pm 1.5$ \\
F.F.F.L. (single) & $-12.0 \pm 4.8$ & $-736.1 \pm 537.3$ & $-791.7 \pm 756.1$ & $14.0 \pm 7.9$ & $21.7 \pm 12.9$ \\
F.F.F.L. (differential) & $-12.1 \pm 5.5$ & $-1083 \pm 536$ & $-8833 \pm 5796$ & $27.3 \pm 5.1$ & $141.6 \pm 105.3$
\end{tabular}

Data are reported as mean \pm 1 S.E.M

parameters of identified system dynamics between subjects. These variances are due to the sensitivity of the pMMS to sensor placements and tension of the sleeve to hold the sensor, which vary $K_{\mathrm{p}}, k_{\mathrm{m} 1}, k_{\mathrm{m} 2}, c_{\mathrm{m}}$ as well as static nonlinearity between the target variable, $\delta \eta$, and the measured pressure, $\delta P_{\mathrm{a}}$.

\section{Model verification of pMMS}

The model verification results are shown in Fig. 4 and given in Table 3. For the joint angle estimation results, normalized root mean square (NRMS) errors were calculated by normalizing the root mean square (RMS) error in the ROM, which is referred to as $e_{\mathrm{J}}$. Meanwhile, because $\Delta \epsilon_{\mathrm{m}}$ is already the normalized variable, the RMS errors of $\Delta \epsilon_{\mathrm{m}}$ were used for the evaluation, which is referred to as $e_{\mathrm{M}}$. Additionally, the correlation coefficient (R-values) for the joint angle $R_{J}$ and muscle fascicle length estimations $\mathrm{R}_{\mathrm{M}}$ are calculated. The $e_{\mathrm{J}}$ and $e_{\mathrm{M}}$ are shown as 4.8 $\pm 0.5 \%$ and $5.7 \pm 1.8 \%$ over the five subjects, respectively. Similarly, the $R_{J}$ and $R_{M}$ indicate strong linearity. The $R_{J}$ and $R_{M}$ are $0.990 \pm 0.002$ and $0.980 \pm 0.014$, respectively. Therefore, while the proposed model may simplify complex muscle-skin-pMMS dynamics, the current pMMS model was able to provide muscle fascicle length and joint angle estimation within 5-6\% NRMS errors, suggesting validity of the proposed model for the motor tasks at different amplitudes and speeds tested here.

\section{External perturbations from surrounding muscles}

The pMMS performance under the external perturbations is shown in Fig. 5 and exhibited in Table 3. Comparing the results of half-amplitude and half-frequency sweep trials, the $e_{\mathrm{J}}$ and $e_{\mathrm{M}}$ of the full-amplitude and fullfrequency sweep trials drastically increased to $13.9 \pm$ $1.7 \%$ and $11.9 \pm 2.0 \%$, respectively. Additionally, the $R_{J}$ and $R_{M}$ decreased to $0.884 \pm 0.036$ and $0.923 \pm 0.016$, respectively. As described in the "Methods" section, these deteriorations of pMMS performance are due to external perturbations from surrounding muscles as well as insensitivity of pMMS outputs to muscular movements around neutral position as shown in Fig. 3.
External perturbation compensation by differential-pMMS The differential-pMMS system was verified by the fullamplitude and frequency sweep trials which demonstrates that the differential-pMMS effectively compensates for the external perturbations (Figs. 3 and 5, Table 3) and improves $e_{\mathrm{J}}$ and $e_{\mathrm{M}}$ to $7.0 \pm 2.5 \%$ and $6.4 \pm 1.2 \%$, respectively. Also, the differential-pMMS estimation results show strong linearity to the target variables which $R_{\mathrm{J}}$ and $R_{M}$ are $0.971 \pm 0.018$ and $0.979 \pm 0.010$, respectively. To further address the affect of differential-pMMS, one-way ANOVA with Tukey-Kramer multiple comparison tests were applied to $e_{\mathrm{J}}, e_{\mathrm{M}}, \mathrm{R}_{\mathrm{J}}$, and $\mathrm{R}_{\mathrm{M}}$ of different pMMS configurations which are shown in Fig. 6. No significant differences were found between single-pMMS halfamplitude and half-frequency (S.H.H.) and differentialpMMS full-amplitude and full-frequency (D.F.F.) trials at all matrices including $e_{\mathrm{J}}, e_{\mathrm{M}}, \mathrm{R}_{\mathrm{J}}$, and $\mathrm{R}_{\mathrm{M}}$. However, significant differences were found between single-pMMS full-amplitude and full-frequency (S.F.F.) and the other two configurations at all matrices. These results indicate that the differential-pMMS configuration is effective in compensating for external perturbations and successfully provides reliable joint angle and muscle fascicle length estimation during the full ROM at the ankle.

\section{Discussion}

Throughout this work, we have investigated the characteristics and capability of the pMMS to provide muscle fascicle length and joint angle estimation. Using silicon tubing (Fig. 2b), the current pMMS design allows for implementation of the electronics outside of an fMRI scanner room, while keeping the air pocket attached to the patient, demonstrating its viability as a MRI-compatible sensor platform. Moreover, because the pMMS can be easily applied to the muscle of interest without being invasively attached to skin, the pMMS provides superior advantages in terms of practicality. Although highorder muscle-skin interaction dynamics were simplified by neglecting 'creep' dynamics, a single pMMS successfully provided comparable estimates to actual measurements of joint angle and muscle fascicle length during 


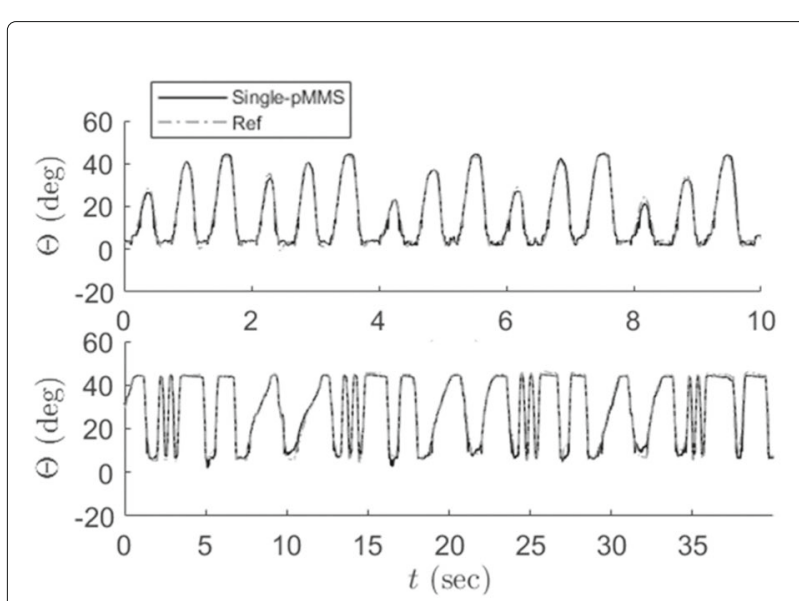

(a)

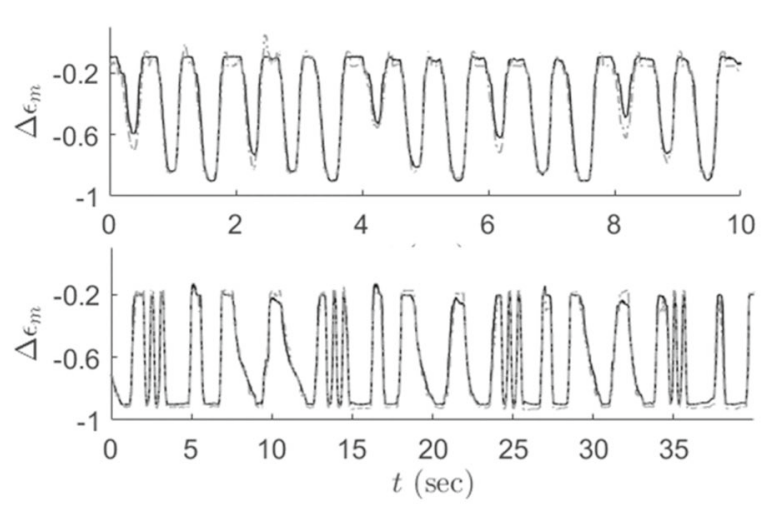

(d)

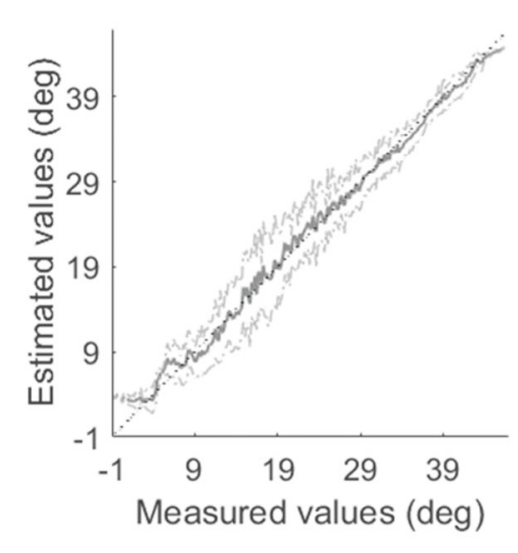

(b)

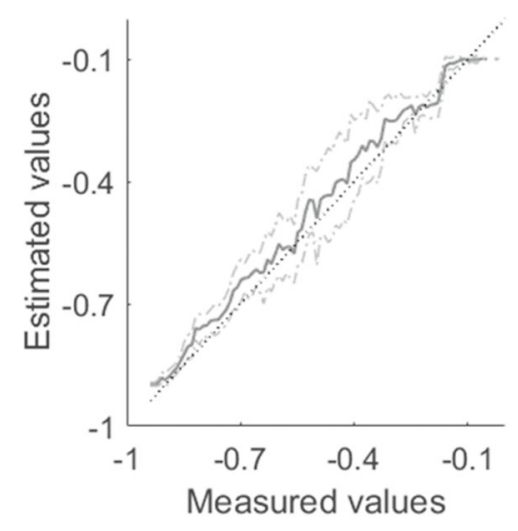

(e)

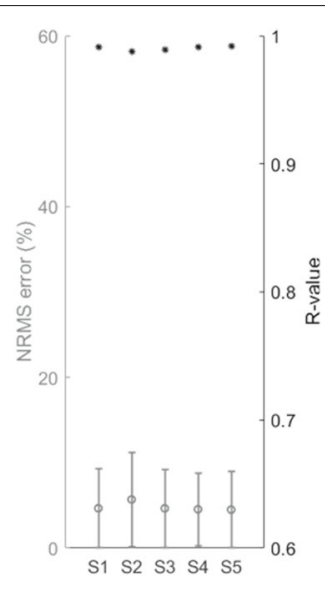

(c)

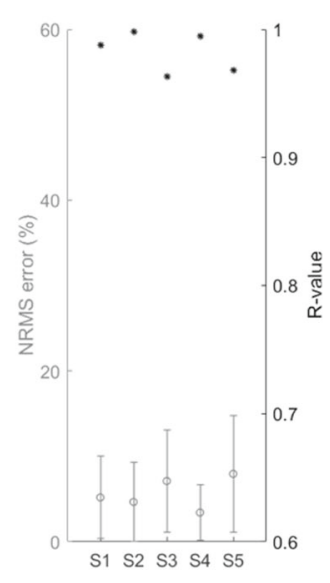

(f)

Fig. 4 Model validation results. a Representative joint angle estimation result from half-amplitude (top) and half-frequency (bottom) sweep trials (S1), b representative linearity plot of joint angle estimation (S1), c summary of NRMS estimation errors (error bars) and R-values (*) of joint angle estimation (S1-S5), d representative muscle fascicle length estimation result from half-amplitude (top) and half-frequency (bottom) sweep trials (S1), e representative linearity plot of muscle fascicle length estimation (S1), $\mathbf{f}$ summary of NRMS estimation errors (error bars) and R-values $(*)$ of muscle fascicle length estimation (S1-S5). The dotted lines and solid lines in the estimation results indicate the measured values and estimation values from pMMS outputs, respectively. For the linearity plots, the solid lines and dotted lines indicate the mean and 1 S.D. of the pMMS linearity, respectively

active dorsiflexion of all five participants within different speeds and amplitudes as shown in Fig. 4. To overcome large estimation errors resulting from perturbation of surrounding muscles during plantar flexion, shown in Fig. 5, we explored the integration of multiple pMMS to provide

Table 3 NRMS errors $\left(e_{\jmath}, e_{M}\right)$ and R-values $\left(R_{J}, R_{M}\right)$ of single-pMMS half-amplitude and half-frequency sweep trials (S.H.H), single-pMMS full-amplitude and full-frequency sweep trials (S.F.F.), and differential-pMMS full-amplitude and full-frequency sweep trials (D.F.F.)

\begin{tabular}{lllll}
\hline Type & $e_{j}$ & $e_{M}$ & $R_{j}$ & $R_{M}$ \\
\hline S.H.H. & $4.8 \pm 0.5 \%$ & $5.7 \pm 1.8 \%$ & $0.990 \pm 0.002$ & $0.980 \pm 0.014$ \\
S.F.F. & $13.9 \pm 1.7 \%$ & $11.9 \pm 2.0 \%$ & $0.884 \pm 0.036$ & $0.923 \pm 0.016$ \\
D.F.F. & $7.0 \pm 2.5 \%$ & $6.4 \pm 1.2 \%$ & $0.971 \pm 0.018$ & $0.979 \pm 0.010$
\end{tabular}

Data are reported as mean \pm 1 S.D. estimates of muscle fascicle length and joint angle. By having one pMMS at both the proximal and distal ends of the TA, we found that the estimated joint angle and muscle fascicle length from differential-pMMS readings were comparable to that of the measurements from the joint encoder and ultrasound imaging, expanding pMMS capacity over a full ROM. Therefore, the pMMS may lead to further understanding of CNS and muscle sensory feedback interactions, as well as enhanced rehabilitative strategies for populations with sensory-motor dysfunction.

Although the current pMMS poses sufficiency of its use in fMRI settings for real-time monitoring of a subject's task performance, more general application usage, such as human motor intent estimation for assistive device control and clinical measurements in rehabilitation 


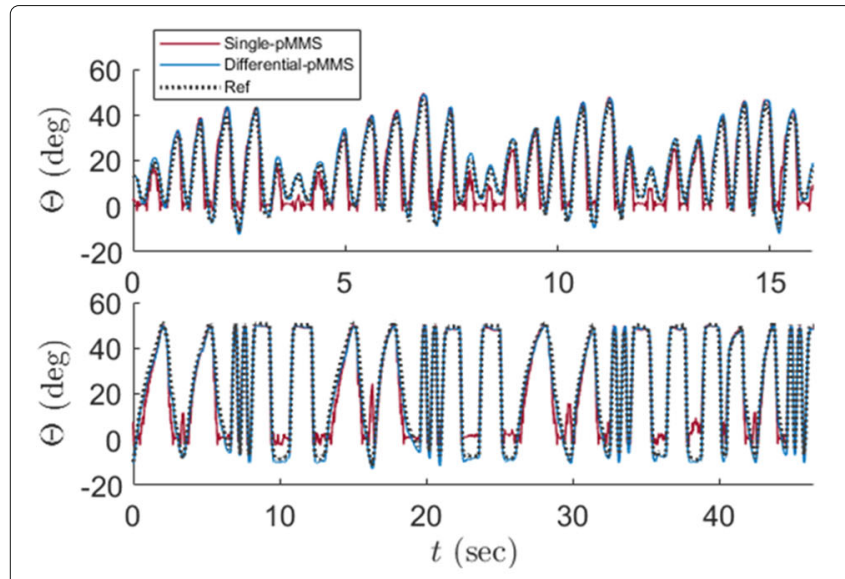

(a)

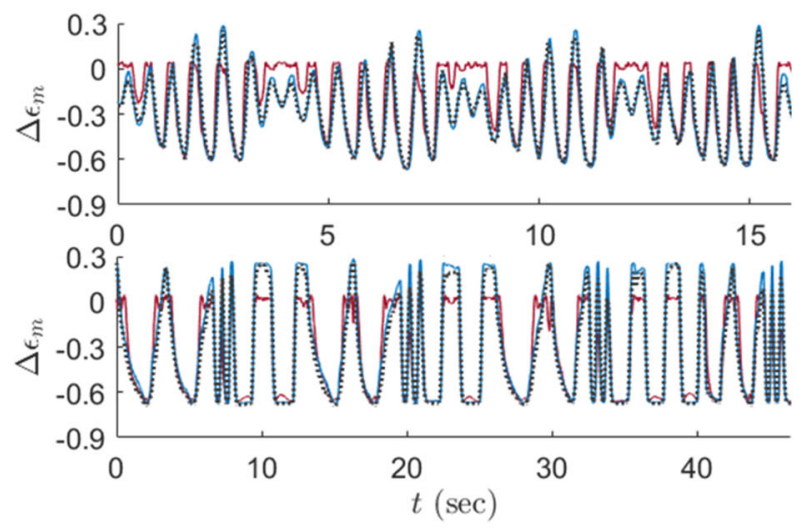

(d)

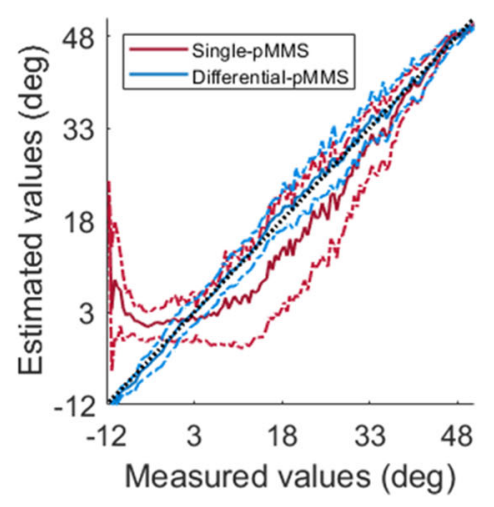

(b)

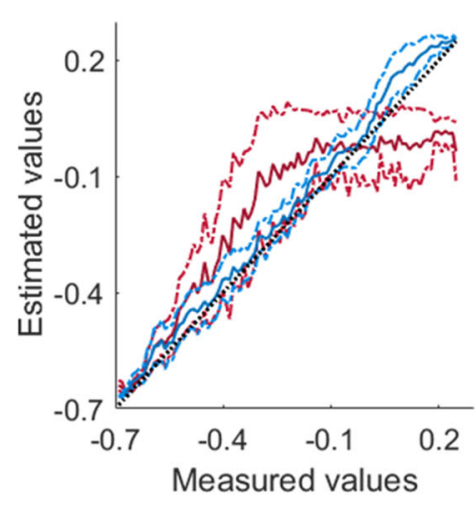

(e)

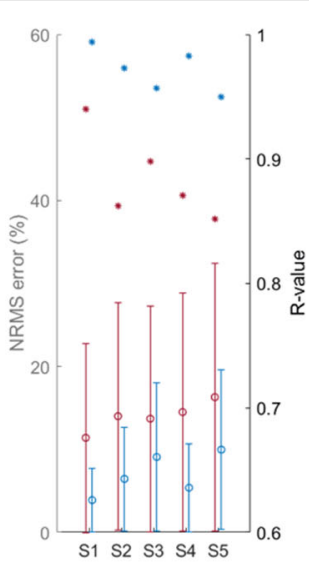

(c)

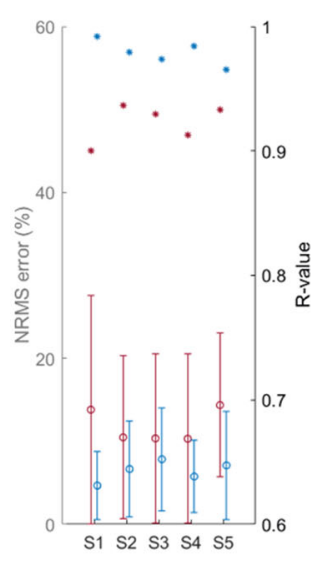

(f)

Fig. 5 Comparison of single-pMMS and differential-pMMS performances for full-amplitude and full-frequency trials. a Representative joint angle estimation result from full-amplitude (top) and full-frequency (bottom) sweep trials (S1), b representative linearity plot of joint angle estimation (S1), c summary of NRMS estimation errors (error bars) and R-values (*) of joint angle estimation (S1-S5), d representative muscle fascicle length estimation result from full-amplitude (top) and full-frequency (bottom) sweep trials (S1), e representative linearity plot of muscle fascicle length estimation (S1), $\mathbf{f}$ summary of NRMS estimation errors (error bars) and R-values $\left(^{*}\right.$ ) of muscle fascicle length estimation. The dotted lines and solid lines in the estimation results indicate the measured values and estimation values from pMMS outputs, respectively. For the linearity plots, the solid lines and dotted lines indicate the mean and 1 S.D. of the pMMS linearity, respectively

settings, needs further investigation. Because muscle mechanical properties are also affected by the level of muscle activation, the current pMMS systems may be limited in providing estimates during isometric contraction or passive stretching movements which is commonly performed in rehabilitation and physical therapy settings. To overcome these potential limitations, the incorporation of EMG data in conjunction with pressure data may be used to enhance the usability of the pMMS outside of fMRI applications. In this scenario, EMG data can be utilized to decouple muscle fascicle length and muscle activity from pressure measures, applying advanced sensor fusion methodologies, e.g. Kalman filter [27], fuzzy logic [28].

\section{Conclusion}

In this work, the pMMS design and associated methodology are proposed. Through several diverse trials, investigating the pMMS's capability to estimate muscle fascicle dynamics under various movement frequencies and joint angle amplitudes, we report high prediction accuracy of joint position and muscle fascicle length. The pMMS poses as a reliable, MRI-safe, wearable sensor for measuring muscle fascicle length and dynamics in populations including persons with amputation or motor dysfunction because of its ability to perform independently from joint angle measurements. The application of the pMMS system in conjunction with fMRI data of brain activity 


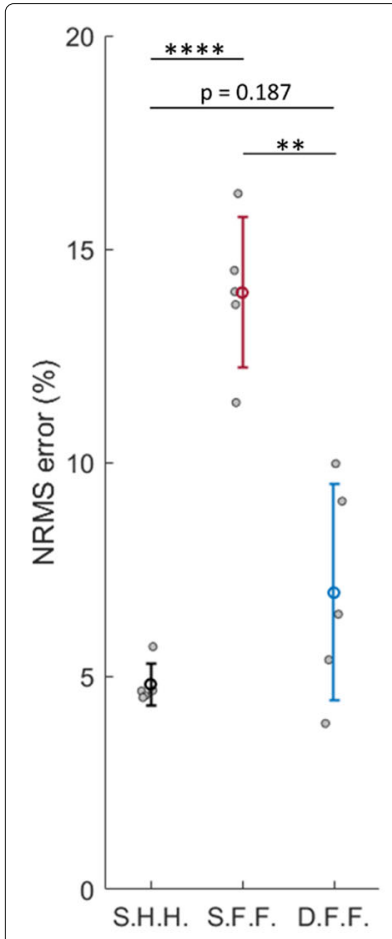

(a)

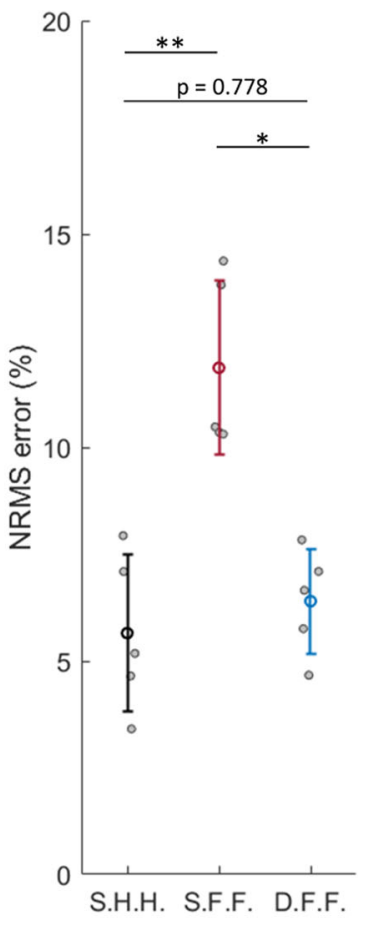

(b)

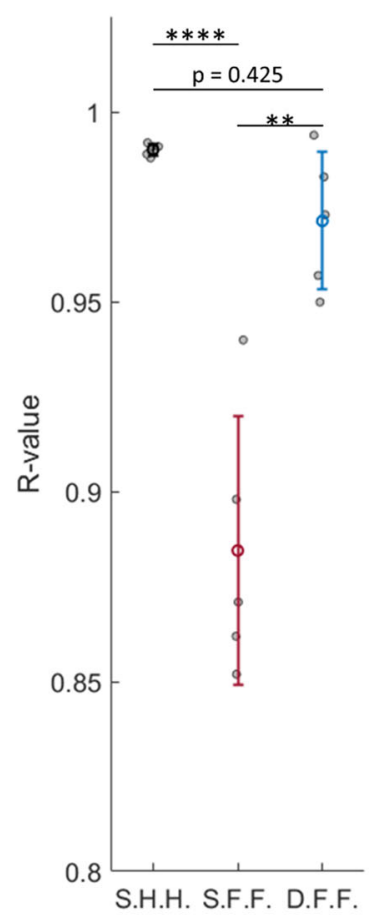

(c)

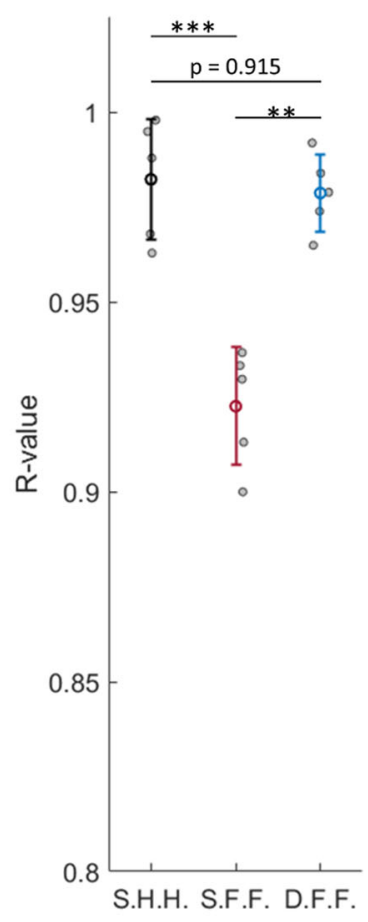

(d)

Fig. 6 Evaluation of single-pMMS and differential-pMMS configurations. $\mathbf{a}$ and $\mathbf{b}$ show NRMS errors of single-pMMS half-amplitude and half-frequency sweep trials (S.H.H), single-pMMS full-amplitude and full-frequency sweep trials (S.F.F.), and differential-pMMS full-amplitude and full-frequency sweep trials (D.F.F.). $\mathbf{c}$ and $\mathbf{d}$ indicate R-value of S.H.H., S.F.F., and D.F.F. Data are reported as mean \pm 1 S.D. Asterisks above bars indicates significant differences between different pMMS configurations. ${ }^{*} p<1 \times 10^{-3}$, ${ }^{* *} p<5 \times 10^{-4},{ }^{* * *} p<1 \times 10^{-4},{ }^{* * *} p<5 \times 10^{-5}$, one-way ANOVA with Tukey-Kramer multiple comparison tests. Where no significance was seen, a $p$ value is shown

may lead to novel discoveries for the augmentation of rehabilitation and mobility. Future work will include the investigation of pMMS usage in non-MRI settings and the incorporation of EMG for more accurate muscle dynamic measures. Additionally, the pMMS will be tested during rehabilitation appropriate tasks such as passive stretching and weight-bearing activity.

\section{Supplementary information}

Supplementary information accompanies this paper at https://doi.org/10.1186/s12984-020-00745-8.

Additional file 1: Supplemental information 1 Identified subject-specific pMMS dynamics. The parameters of identified linear system dynamics and static non-linearity are reported. Supplemental information 2 Joint angle and muscle fascicle length estimation results of all five participants. The estimation results of joint angle and muscle fascicle length of all five participants are included. NRMS error and R-value of joint angle and muscle fascicle length estimation results are reported.

\section{Abbreviations}

fMRI: Functional Magnetic Resonance Imaging; MRI: Magnetic Resonance Imaging; pMMS: Pressure-based Muscular Motion Sensor; TA: Tibialis Anterior; sEMG: Surface Electromyography; FMG: Forcemyography; FSR: Force Sensitive Registers; MIT: Massachusetts Institute of Technology; COUHES: Committee on the Use of Humans as Experimental Subjects; ROM: Range Of Motion; PL: Peroneus Longus; TP: Tibialis Posterior; GAS: Gastrocnemius; NRMS: Normalized Root Mean Square; RMS: Root Mean Square
Acknowledgements

We thank Peter Calvaresi for assistance with ultrasound imaging

\section{Authors' contributions}

HS developed pMMS, computational model, performed experiment design, data collection, analysis, and writing of the manuscript. El contributed to experiment design, data collection, analysis, and writing of the manuscript. SS assisted with data collection, analysis, and manuscript editing. $\mathrm{HH}$ contributed to the funding, experiment design, provision of resources and writing.

\section{Funding}

This work was funded by the MIT Media Lab Consortium.

\section{Availability of data and materials}

All data are available in the main text or the supplemental information. Any code used for the processing of data in this manuscript can be obtained from the authors upon reasonable request.

\section{Ethics approval and consent to participate}

All experiments were carried out with informed consent at the Massachusetts Institute of Technology (MIT), under the approval of the Committee on the Use of Humans as Experimental Subjects (COUHES).

\section{Consent for publication}

Consent for publication were obtained from all participants of the current study.

\section{Competing interests}

The authors declare that they have no competing interests.

\section{Author details}

${ }^{1}$ Center for Extreme Bionics, Massachusetts Institute of Technology (MIT) Media Lab, 02139 Cambridge, MA, USA. ${ }^{2}$ Harvard-MIT Division of Health 
Sciences and Technology, MIT, 02139 Cambridge, MA, USA. ${ }^{3}$ Department of Media Arts and Sciences, MIT, 02139 Cambridge, MA, USA.

Received: 3 March 2020 Accepted: 5 August 2020

Published online: 26 August 2020

\section{References}

1. Huettel SA. Event-related fmri in cognition. Nueroimage. 2012;62(2): 1152-6.

2. Ehrsson H, Fagergren A, Jonsson $T$, Westling $G$, Johansson R, Forssberg $\mathrm{H}$. Cortical activity in precision-versus power-grip tasks: an fmri study. J Neurophysiol. 2000;83(1):528-36.

3. Mehta J, Verber M, Wieser J, Schmit B, Schindler-Ivens S. A novel technique for examining human brain activity associated with pedaling using fmri. J Neurosci Methods. 2009;179(2):230-9.

4. Elhawary H, Zivanovic A, Rea M, Davies B, Besant C, McRobbie D, Desouza N, Young I, Lamperth M. A modular approach to mri-compatible robotics. IEEE Eng Med Biol Mag. 2008;27(3):35-41.

5. Tsekos N, Christoforou E, Ozcan A. A general-purpose mr-compatible robotic system: Implementation and image guidance for performing minimally invasive interventions. IEEE Eng Med Biol Mag. 2008;27(3):51-8.

6. Hidler J, Hodics T, Xu B, Dobkin B, Cohen L. Mr compatible force sensing system for real-time monitoring of wrist moments during fmri testing. J Neurosci Methods. 2006;155(2):300-7.

7. Tada M, Kanade T. Design of an mr-compatible three-axis force sensor. In: Proc. IEEE/RSJ Int. Conf. Intell. Robots Syst. (IROS). Edmonton: IEEE; 2005. p. 3505-10.

8. Chapuis D, Gassert R, Sache L, Burdet E, Bleuler H. Design of a simple mri/fmri compatible force/torque sensor. In: Proc. IEEE/RSJ Int. Conf. Intell. Robots Syst. (IROS). Sendai: IEEE; 2004. p. 2593-9.

9. Vigaru B, Sulzer J, Gassert R. Design and evaluation of a cable-driven fmri-compatible haptic interface to investigate precision grip control. IEEE Trans Haptics. 2016;9(1):20-32.

10. Rossignol S, Dubuc R, Gossard J. Dynamic sensorimotor interactions in locomotion. Physiol Rev. 2006;86:89-154.

11. Kroger S. Proprioceptoin 2.0: novel functions for muscle spindles. Neuromuscul Disord. 2018;31(5):593-8.

12. Biewener AA, Roberts TJ. Muscle and tendon contributions to force, work, and elastic energy savings: a comparative perspective. Exerc Sport Sci Rev. 2000;28(3):99-107.

13. Taylor CR, Abramson HG, Herr H. Low-latency tracking of multiple permanent magnets. IEEE Sensors J. 2019;19(23):11458-68.

14. Sikdar S, Wei Q, Cortes N. Dynamic ultrasound imaging applications to quantify musculoskeletal function. Exerc Sport Sci Rev. 2014;42(3):126-35.

15. DeJong AF, Mangum LC, Hertel J. Gluteus medius activity during gait is altered in individuals with chronic ankle instability: An ultrasound imaging study. J Gaitpost. 2019;71:7-13.

16. Franchi MV, Raiteri BJ, Longo S, Sinha S, Narici MV, Csapo R. Muscle architecture assessment: strengths, shortcomings and new frontiers of in vivo imaging techniques. J Ultrasmedbio. 2018;44(12):2492-504.

17. Bae G, Song J, Kim B. Imitation of human motion based on variable stiffness actuator and muscle stiffness sensor. In: Proc. IEEE/ASME Int. Conf. Intell. Mechatron. (AIM). Wollongong: IEEE; 2013. p. 1016-1020.

18. Bo Z, Sundholm M, Cheng J, Cruz H, Lukowicz P. Measuring muscle activities during gym exercises with textile pressure mapping sensors. Pervasive Mob Comput. 2017;38:331-45.

19. Belbasis A, Fuss FK. Muscle performance investigated with a novel smart compression garment based on pressure sensor force myography and its validation against emg. Front Physiol. 2018;9:1-13.

20. Huxley HE. The mechanism of muscular contraction. Science. 1969;164(3886):1356-65.

21. Narici M, Binzoni T, Hiltbrand E, Fasel J, Terrier F, Cerretelli P. In vivo human gastrocnemius architecture with changing joint angle at rest and during graded isometric contraction. J Physiol. 1996;496(1):287-97.

22. Kong K, Tomizuka M. A gait monitoring system based on air pressure sensors embedded in a shoe. IEEE/ASME Trans Mechatron. 2009;14(3): 358-70.

23. Dowling NE. Mechanical behavior of materials: engineering methods for deformation, fracture, and fatigue. 2nd. Englewood Cliffs: Pearson-Prentice Hall; 1999.
24. Boyer G, Laquieze L, Le Bot A, Laquieze S, Zahouani H. Dynamic indentation on human skin in vivo: ageing effects. Skin Res Technol. 2009;15(1):55-67.

25. Khatyr F, Imberdis C, Vescovo P, Varchon D, Lagarde J. Model of the viscoelastic behaviour of skin in vivo and study of anisotropy. Skin Res Technol. 2004;10(2):96-103.

26. Hunter I, Korenberg M. The identification of nonlinear biological systems: Wiener and hammerstein cascade models. Biol Cybern. 1986;55(2-3): 135-44.

27. Sun S, Deng Z. Multi-sensor optimal information fusion kalman filter. Automatica. 2004;40(6):1017-23.

28. Stover J, Hall D, Gibson R. A fuzzy-logic architecture for autonomous multisensor data fusion. IEEE Trans Ind Electron. 1996;43(3):1017-23.

\section{Publisher's Note}

Springer Nature remains neutral with regard to jurisdictional claims in published maps and institutional affiliations.
Ready to submit your research? Choose BMC and benefit from:

- fast, convenient online submission

- thorough peer review by experienced researchers in your field

- rapid publication on acceptance

- support for research data, including large and complex data types

- gold Open Access which fosters wider collaboration and increased citations

- maximum visibility for your research: over $100 \mathrm{M}$ website views per year

At BMC, research is always in progress.

Learn more biomedcentral.com/submissions 